\title{
Modelado de un sistema consciente del contexto para soportar intervenciones en actividad física y nutrición saludable
}

\author{
Katherine Xiomar González* \\ Miguel Ángel Carvajal** \\ Gineth Magaly Cerón*** \\ Diego Mauricio López ${ }^{* * * *}$
}

\author{
Recibido: 30/07/2015 • Aceptado: 11/12/2015 \\ DOl: $10.22395 /$ rium.v15n28a9
}

\begin{abstract}
Resumen
Los sistemas de salud en todo el mundo afrontan el desafío de combatir el gran incremento de enfermedades crónicas no transmisibles (ECNT), especialmente enfermedades cardiovasculares. Estas enfermedades pueden ser prevenidas si se logra la adopción de hábitos y estilos de vida saludables por parte de las personas, fundamentalmente el incremento de la actividad física y la nutrición sana. El objetivo de este artículo es describir el proceso de modelado de un sistema consciente del contexto, con el fin de soportar intervenciones que promuevan la actividad física y la dieta saludable, y se adapten a las características del contexto del usuario. Los principales resultados de este trabajo son: a) un modelo de clasificación del contexto en salud y un modelo del proceso de adaptabilidad y personalización del contexto, b) una propuesta de un modelo de contexto para un sistema consciente en el contexto que apoye la promoción de actividad física y nutrición saludable, c) una arquitectura de referencia, y un prototipo del sistema desarrollado sobre esta arquitectura, el cual consiste en una aplicación móvil soportada en tecnologías NFC y GPS, y d) la evaluación de la usabilidad de la solución.
\end{abstract}

Palabras clave: sistema consciente del contexto, modelo del contexto,

* Estudiante de Ingeniería Electrónica y Telecomunicaciones de la Universidad del Cauca, Popayán, Colombia. Investigador Asociado del Grupo de Ingeniería Telemática, Línea de Servicios en Salud. Dirección: Calle 5 N.4-70. Popayán, Colombia. Tel.+57(8) 209800 Ext. 2129. E-mail: kgonzalez@unicauca.edu.co

** Estudiante de Ingeniería Electrónica y Telecomunicaciones de la Universidad del Cauca, Popayán, Colombia. Investigador Asociado del Grupo de Ingeniería Telemática, Línea de Servicios de Salud. Dirección: Calle 5 N. 4 - 70. Popayán, Colombia. Tel. +57(8) 209800 Ext. 2129. E-mail: miguelpalacios @unicauca.edu.co

*** PhD (c) en Ingeniería Telemática, Investigador Asociado del Grupo de Ingeniería Telemática, Línea de Servicios de Salud, Universidad del Cauca. Dirección: Calle 5 N.․ 4 - 70. Popayán, Colombia. Tel. +57(8) 209800 Ext. 2175. E-mail: gceron@unicauca.edu.co

**** PhD en Ciencias Biomédicas, Profesor Titular y Coordinador de la Línea de Investigación de Servicios en Salud, Universidad del Cauca. Dirección: Calle 5 N. ${ }^{\circ}$ - 70. Popayán, Colombia. Tel. +57(8) 209800 Ext. 2175. E-mail: dmlopez@unicauca.edu.co 
datos del contexto, NFC, GPS

\title{
Modeling of a context-aware system to support interventions in physical activities and healthy nutrition
}

\begin{abstract}
Health systems around the world are currently facing a challenge to fight the tremendous growth of non-transmissible chronic diseases (NTCD), especially cardiovascular diseases. These diseases can be prevented if adoption of healthy habits and lifestyles are adopted by people, specifically by increasing their physical activity and having a healthy nutrition. The objective of this article is to describe the modeling process of a contextaware system with the purpose of supporting interventions to promote physical activity and healthy nutrition, duly adjusted to the characteristics of the user's context. The main results of this article are: a) a classification model of health context and a context adaptability and personalization process model; b) a proposal of a context model for a context-aware system, which supports the promotion of physical activity and healthy nutrition; c) a reference architecture and a prototype of the system developed on such architecture, which consists of a mobile application supported by NFC and GPS technologies; and d) an evaluation of the solution usability.
\end{abstract}

Key words: context-aware system; context model; context data; NFC; GPS. 


\section{INTRODUCCIÓN}

Los sistemas de salud en todo el mundo están experimentando uno de los mayores desafíos de este siglo: el continuo incremento en las tasas de morbilidad y mortalidad causado por enfermedades crónicas no transmisibles (ECNT) [1]. Dentro de las ECNT más prevalentes se encuentran las enfermedades cardiovasculares que provocan aproximadamente 17,3 millones de muertes cada año, seguidas del cáncer con 7,6 millones de defunciones, las enfermedades respiratorias con 4,2 millones y la diabetes con 1,3 millones de muertes al año [1].

Para combatir las ECNT, la Organización Mundial de la Salud (OMS) ha establecido un plan de acción para reducir su alta prevalencia, el cual está orientado al diseño de intervenciones eficaces que aborden factores de riesgo comunes, como son la dieta malsana, la inactividad física, el consumo de tabaco y el uso nocivo del alcohol [2]. Dado el carácter comportamental de los anteriores factores de riesgo, estos pueden ser abordados mediante estrategias educativas y de cambio de comportamiento, que promuevan la adopción de hábitos y estilos de vida saludables por parte de los individuos [3]. Además, estas estrategias se deben orientar de manera individual para lograr un impacto positivo en la salud cardiovascular [4].

Considerando la mayor prevalencia de las enfermedades cardiovasculares y de la diabetes, el enfoque de este trabajo es la promoción de la actividad física y de la nutrición saludable. Tradicionalmente se han utilizado como estrategias y mecanismos de promoción los folletos, charlas, vídeos, llamadas telefónicas y consejería personalizada. Sin embargo, acorde con las tendencias mundiales, las intervenciones son efectuadas cada vez usando estrategias innovadoras, entre las que se encuentra el uso de las tecnologías de información y comunicación (TIC) [5].

En [6] se realizó una revisión sistemática de las principales intervenciones soportadas en TIC para la promoción de hábitos y estilos de vida saludables. Se encontró que las intervenciones existentes son principalmente aplicaciones web que soportan intervenciones para educar y promover cambios de comportamiento en los individuos. Sin embargo, existe una tendencia reciente a usar aplicaciones móviles (Apps), teléfonos celulares, sensores de actividad física, monitores de ritmo cardiaco, GPS, Kinect, televisores inteligentes, entre otras tecnologías, para soportar la entrega y captura de información de las personas. Otros trabajos están enfocados en promover el cambio de comportamiento de las personas y mejorar la experiencia (satisfacción) del usuario. Todas estas iniciativas demuestran la necesidad de personalización y más concretamente la adaptación de las intervenciones TIC a las necesidades y características de los usuarios. 
Los sistemas adaptativos ofrecen una solución a este desafío de personalización y adaptación, permitiendo que los sistemas se adapten a los usuarios y no los usuarios al sistema. Los sistemas adaptativos tienen la capacidad de cambiar y aprender de la experiencia, además de adaptarse al comportamiento del usuario haciendo uso de modelos de usuario detallados, que incluyen sus objetivos, intereses y preferencias, y donde el contexto del usuario, también conocido como modelo de contexto tiene un rol fundamental. Estos sistemas reciben también el nombre de sistemas conscientes en el contexto [7-10]. En [7-8, 11] se señala la definición de Dey como la más apropiada para entender qué es el contexto: "el contexto es cualquier información que puede ser usada para caracterizar la situación de una entidad, que puede ser un usuario, un lugar o un objeto, y que es relevante para la interacción entre el usuario y un sistema, incluyendo usuarios y sus propias aplicaciones". En [12-13] se menciona que los sistemas conscientes del contexto pueden ser también sistemas de recomendación, los cuales son conocidos como CARS (sistemas de recomendación conscientes en el contexto).

Actualmente existen sistemas personalizados para promoción de hábitos saludables, principalmente para la actividad física. Sin embargo, estos solo se desarrollan para ambientes muy específicos y no ofrecen un marco para la realización del modelo de contexto [14]. En [11] se encontraron marcos para la caracterización del contexto y el desarrollo de aplicaciones conscientes en el contexto en el área de la salud. Sin embargo, en ninguno de los sistemas se menciona cómo realizar un modelo del contexto, ni cómo realizar el modelo de datos teniendo en cuenta únicamente datos del contexto y no ítems de calificación del usuario para entregar recomendaciones. En [15], se evidencia la importancia de modelar ordenadamente la información del usuario y su contexto y de implementar el sistema consciente en el contexto con base en un modelo organizado de datos. También se definen algunos modelos, y se describe un modelo de contexto como aquel que define información inteligente y reglas de adaptación predefinida que permiten adaptar la ejecución lógica de las aplicaciones. Sin embargo, no se definen modelos en el área de la salud ni frameworks para construirlos, por lo cual se hace necesario adaptarlos a la salud en temas de promoción.

Considerando los problemas descritos anteriormente, en este artículo se propone modelar un sistema consciente en el contexto que contribuya a la promoción actividad física y nutrición saludable, a través de la recomendación de diferentes intervenciones TIC que hayan sido evaluadas por profesionales en salud. Este artículo se estructura así: primero se realiza una revisión de los trabajos relacionados con este tema; posteriormente se describe la metodología a seguir en la investigación, seguida de la sección de resultados, que fue dividida en tres subsecciones: a) un modelo de clasificación del contexto en salud y un modelo del proceso de adaptabilidad y personalización del contexto, b) una propuesta de un modelo de contexto para el sistema consciente en el 
contexto como apoyo a la promoción de actividad física y nutrición saludable, c) una arquitectura de referencia para implementar el sistema y la descripción del prototipo del sistema desarrollado, incluyendo una evaluación de usabilidad. Finalmente se presentan las recomendaciones y conclusiones del trabajo.

\section{METODOLOGÍA}

En la figura 1, se observa la metodología de investigación que se siguió en el desarrollo de este trabajo [16]. Se inicia con una base conceptual a partir de una revisión sistemática de temas relacionados; luego se seleccionaron las tecnologías para sensar los datos del contexto, y tecnologías para los desarrollos software, usando la metodología Delphi [17]. Para el diseño, desarrollo y evaluación del prototipo del sistema se adoptaron algunos principios de la metodología de diseño centrado en el usuario (DCU) [18], y se realizaron pruebas de usabilidad con un grupo de usuarios que evaluaron el prototipo presentado en este artículo.

Para personalizar un sistema consciente en el contexto se requiere tener un modelo organizado con información personal de cada usuario incluyendo sus gustos y preferencias, además de datos tomados del contexto del usuario de manera no invasiva y que se relacionan con su salud y sus diferentes roles [19]. Esta información puede ser estructurada a través de 5 preguntas llamadas "las 5ws" [7], los modelos de contexto jerárquicos $[9,20]$, modelo de proceso de contexto adaptativo [20] o consulta con expertos. Estos principios fueron adaptados en un modelo de procesos propio para el diseño del modelo de contexto del sistema consciente de contexto para la promoción de la actividad física y la dieta saludable, el cual es descrito en detalle en la sección de resultados.

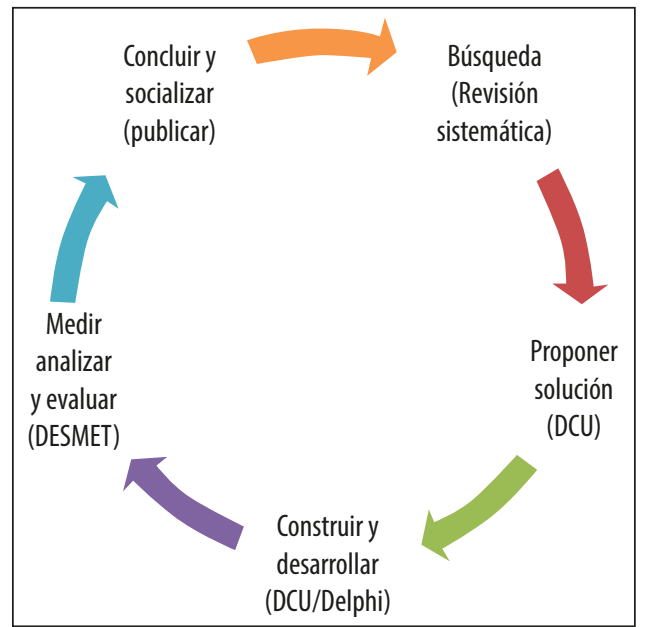

Figura 1. Metodología de investigación para ingeniería.

Fuente: elaboración propia 


\section{RESULTADOS}

Los resultados de este trabajo se han organizado de la siguiente manera: a) Una adaptación teórica de diferentes frameworks para la implementación del modelo de contexto en el área de salud, b) propuesta del modelo de contexto para el sistema consciente en el contexto, c) arquitectura, prototipo y evaluación de usabilidad del sistema desarrollados con el fin de demonstrar la aplicabilidad del modelo propuesto.

\subsection{Frameworks para un modelo de contexto en el área de salud}

\subsubsection{Modelo de clasificación del contexto en salud}

La figura 2 representa la definición del contexto propuesta en este trabajo, la cual es una clasificación de 4 capas para contexto en salud que experimenta un usuario. Este modelo nace a partir de la definición del contexto mencionada en [8], donde se hace referencia puntual a los lugares donde se encuentra el usuario, las actividades que realiza y las personas y objetos que lo rodean [7, 9, 21]. Estos elementos se tomaron como referencia para generar un modelo en cebolla, donde se agrupan los diferentes contextos que influyen en la salud de las personas: contexto digital (dispositivos que tiene el usuario), contexto personal (¿quién es? y ¿por qué usa el sistema?), contexto físico (¿qué hace?, ¿dónde está?) y factores de riesgo en salud que son cualquier rasgo, característica o exposición de un individuo que aumente su probabilidad de sufrir una enfermedad o lesión [22].

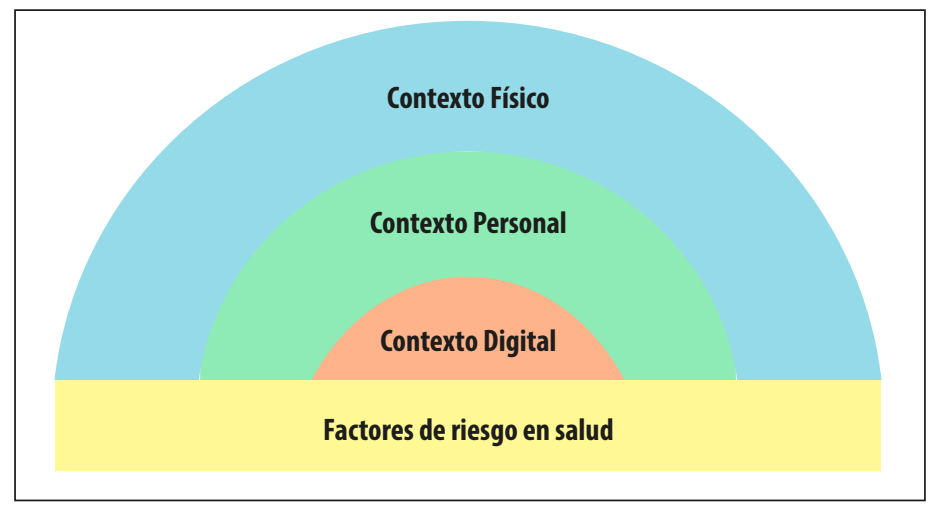

Figura 2. Modelo de clasificación del contexto en salud.

Fuente: elaboración propia

\subsubsection{Modelo del proceso de la adaptabilidad y personalización del contexto}

Ya que el contexto contempla muchas características del usuario, se hace difícil que el sistema solicite todos estos datos al usuario de manera manual. Por esta razón se sugiere modelar la toma de datos, la clasificación y la adaptación de dichos datos, para 
presentarle la mejor respuesta que el usuario desea tener. Se realizó una adaptación del modelo del proceso de adaptabilidad del contexto [20] con el fin de obtener una serie de pasos como guía en la construcción del modelo del contexto en el área de salud. La adaptación consta de 4 etapas y toma el nombre de Modelo del proceso de la adaptabilidad y personalización del contexto, como se observa en la figura 3.

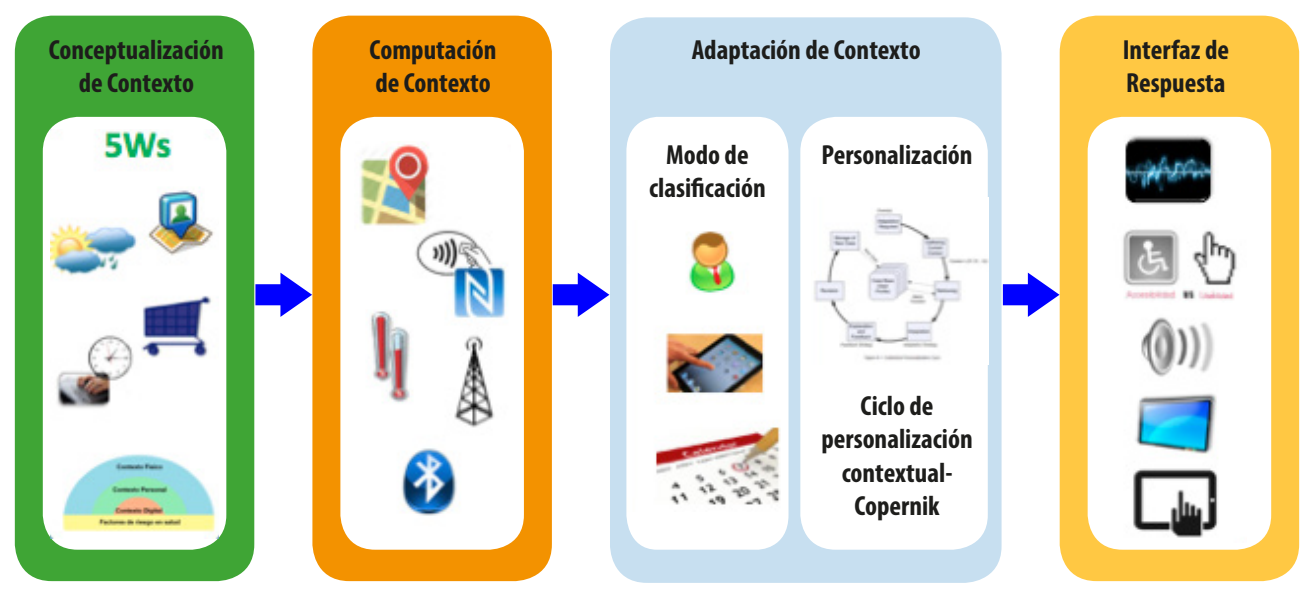

Figura 3. Modelo del proceso de la adaptabilidad y personalización del contexto.

Fuente: elaboración propia

Las cuatro etapas de los modelos se describen a continuación:

Primera etapa, conceptualización del contexto [7, 21]. Esta etapa se caracteriza utilizando el método de las 5WS [7] y la definición y selección de datos relevantes para el sistema, como ubicación, temperatura, actividades, etc. [19]; un modelo de clasificación jerárquico [9], y el modelo de clasificación del contexto de la figura 2.

Segunda etapa, computación del contexto. Aquí se debe realizar la selección de la tecnología para sensar el contexto, la detección o el sensado de los datos que se determinaron en la primera fase, su procesamiento y su almacenamiento. Esto se hace con base en lo propuesto por [19] y se realiza mediante una comparación de tecnologías para seleccionar la mejor.

Tercera etapa, adaptación de contexto. Esta se organizó en dos partes: la primera incluye el modo de clasificación: aquí se hace referencia a un proceso manual de adaptación, es decir, que el sistema antes de realizar una acción pregunta lo que el usuario desea. La segunda parte corresponde a la personalización: esta etapa se refiere al proceso automático en que el sistema aprende y realiza acciones en el momento apropiado 
cuando el usuario quiera que ocurran, para esto se usa el marco de referencia llamado Ciclo de personalización contextual Copernik [23].

La última etapa hace referencia a cómo toda la información va a ser mostrada al usuario, teniendo en cuenta: facilidades de uso, accesibilidad, problemas de discapacidad y que sea amigable al usuario. En esta etapa se usa la metodología del diseño centrado en el usuario DCU [18]. Esta parte será mostrada más adelante con imágenes del funcionamiento del prototipo del sistema.

\subsection{Modelo del contexto}

Basados en el framework conceptual, y siguiendo las etapas del modelo de proceso, se propone el modelo de contexto del sistema consciente de contexto para la promoción de actividad física y dieta saludable. Este modelo de contexto contiene los datos seleccionados para el sistema y las relaciones entre ellos. Con base en esto datos se infiere nueva información y se realizan las recomendaciones y personalización basada en el contexto. El modelo del contexto consta de tres partes: selección de datos, modelado de relaciones y representación gráfica con recomendaciones. A continuación, se analiza cada una de ellas.

\subsubsection{Selección de datos}

Se deben definir los datos del contexto que serán tomados por el sistema, ya sea solicitándolos al usuario directamente o tomándolos con dispositivos externos como NFC (Near Field Communication), GPS (Global Positioning System), entre otros. Primero se hace la clasificación y selección de datos según las 5 Ws y después se relacionan los datos.

\section{- Clasificación según las 5 W}

Los datos del contexto se clasifican en 5 grupos, de acuerdo a las 5W [7]. En la tabla 1 se muestra la clasificación de los datos que es necesarios obtener, para que el sistema pueda recomendar al usuario la mejor intervención TIC. Para este artículo se tienen en cuenta los datos asociados a actividad física y nutrición saludable; pero la figura puede ser usada para cualquier tópico en el área de la salud. 
Tabla 1. Clasificación de datos del contexto de acuerdo con las 5Ws

\begin{tabular}{|c|l|l|l|c|}
\hline \multicolumn{1}{|c|}{ ¿Quién? } & \multicolumn{1}{|c|}{ ¿Dónde? } & ¿Qué? & ¿Cuándo? & ¿Por qué? \\
\hline $\begin{array}{l}\text { Datos de usuario: } \\
\text { peso, ritmo cardia- } \\
\text { co, etc. }\end{array}$ & $\begin{array}{l}\text { Localización general: casa, } \\
\text { trabajo, universidad, etc. }\end{array}$ & $\begin{array}{l}\text { Actividades: } \\
\text { cocinar, tra- } \\
\text { bajar, etc. }\end{array}$ & $\begin{array}{l}\text { Tiempo/ tempora- } \\
\text { da: vacaciones, fes- } \\
\text { tivos, laboral. }\end{array}$ & $\begin{array}{l}\text { Razones / de- } \\
\text { seos: bajar de } \\
\text { peso }\end{array}$ \\
\hline $\begin{array}{l}\text { Personas cercanas: } \\
\text { compañeros de tra- } \\
\text { bajo, hijos, etc. }\end{array}$ & $\begin{array}{l}\text { Localización específica: } \\
\text { cocina, dormitorio, ofici- } \\
\text { na, etc. }\end{array}$ & - & - & - \\
\hline- & $\begin{array}{l}\text { Características de los lu- } \\
\text { gares: temperatura, clima, } \\
\text { etc. }\end{array}$ & - & - & - \\
\hline
\end{tabular}

Fuente: elaboración propia

\section{- Tablas de relaciones entre datos}

Una vez definidos los datos, se identifican las relaciones entre ellos. Con estos dos elementos (datos y relaciones), se procede a realizar el modelado de la información del sistema consciente en el contexto.

Los datos seleccionados para el sistema se organizan en la tabla 2, desde V1 hasta $\mathrm{Vn}$, donde $\mathrm{n}$ representa el número total de datos seleccionados, que son datos genéricos del contexto del usuario. Para este artículo se tomará un ejemplo de las múltiples relaciones que se tendrán en el sistema:

Ejemplo: V1: ubicación específica cocina, V2: ubicación específica lugar recreativo, V3: estar acompañado V4: temperatura, V5: ubicación, V6: Tiempo disponible.

La tabla 2, contiene los datos seleccionados del contexto y las relaciones entre ellos.

Tabla 2. Relación de datos y clasificación

\begin{tabular}{|c|c|c|c|c|}
\hline Datos & $V 1$ & $V 2$ & $V 3$ & $\ldots$ \\
\hline V1 & Relación V1 - V1 & Relación V1 - V2 & Relación V1 - V3 & $\ldots$ \\
\hline V2 & Relación V2 - V1 & Relación V2 - V2 & Relación V2 - V3 & $\ldots$ \\
\hline V3 & Relación V3 - V1 & Relación V3 - V2 & Relación V3 - V3 & $\ldots$ \\
\hline$\ldots$ & $\ldots$ & $\ldots$ & $\ldots$ & $\ldots$ \\
\hline
\end{tabular}

Fuente: elaboración propia 
En la tabla 2, la diagonal (casillas en azul) representa la relación de una variable con sí misma. Estas no son relevantes para este modelo, por tal razón no se tendrán en cuenta al modelar el contexto. Las relaciones que se encuentran de color naranja, por debajo de la diagonal azul, son las relaciones significativas a modelar porque relaciona distintos datos del contexto, permitiendo garantizar una mejor inferencia y con ello una mejor recomendación por parte del sistema. Las relaciones pueden ser de tres tipos:

Sin relación: los datos son totalmente independientes, como es el caso de la relación V1-V2; en el primer caso, el sistema infiere que quiere cocinar o que el usuario va a comer; para esta situación se recomendaría nutrición saludable, mientras que en el otro caso, en un espacio recreativo, la intervención sería de ejercicio.

Complementaria: se presenta cuando la información de dos datos es diferente, pero ambas son relevantes para una recomendación. Su información es un valor agregado de la otra, como V2 y V3. En este caso los datos poseen una relación complementaria; de esta manera, la intervención TIC sería un ejercicio para realizar en grupo, dado que V3 indica que el usuario está acompañado en ese momento.

Dependencia: una variable no puede ser obtenida sin la presencia de la otra, como el caso de un sistema que tome la edad a partir de la fecha de nacimiento.

Las relaciones también dependen de cómo se recolectan los datos. Ejemplo: si se va a sensar la temperatura con termómetro y la ubicación por GPS; su relación es complementaria; sin embargo, si no fuera tomada la temperatura con termómetro sino a partir de la ubicación, como en el caso del reporte del clima, por ejemplo "Nueva York 22 grados Celsius", en este caso será dependiente la temperatura con la localización GPS.

\section{- Representación gráfica de relaciones y recomendaciones}

Siguiendo la guía de la figura 3, para construir el modelo de contexto, se identifican las relaciones de los datos y de acuerdo con esto las recomendaciones, que serán las intervenciones TIC ya sea de ejercicio (ocio, ejercicio físico, y pausas activas) o de nutrición saludable.

En la figura 4 se presenta la manera de inferir recomendaciones, teniendo en cuenta las relaciones entre datos y considerando las posibles combinaciones de sus valores. 


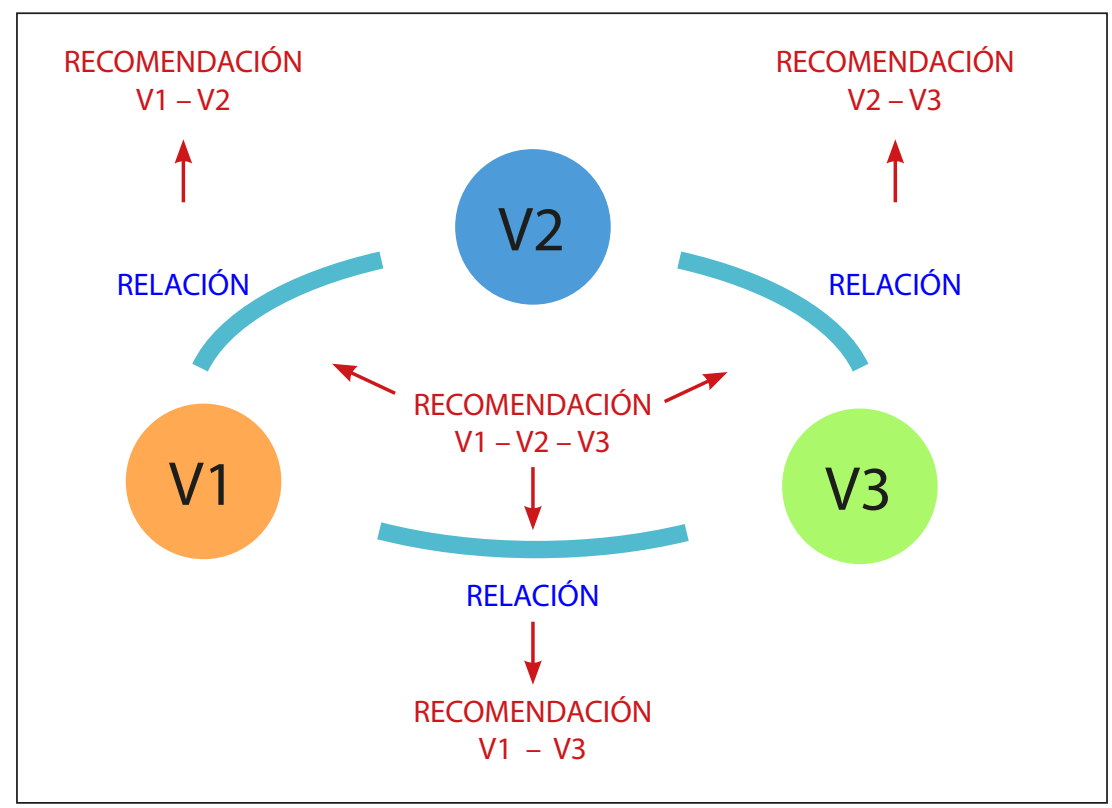

Figura 4. Diagrama de recomendaciones con base en relaciones.

Fuente: elaboración propia

Ejemplo: Se definen relaciones complementarias, es decir, que los tres datos pueden tenerse en cuenta dentro de una recomendación y que la toma de los datos es independiente. La posible recomendación es:

V1: Lugar cerrado - V2: temperatura baja - V3: 20 min (temperatura, ubicación y tiempo disponible).

Se deben realizar dos procesos: el primero es clasificar las intervenciones TIC que no le requieran al usuario un tiempo mayor de 20 minutos de duración, haciendo uso de los metadatos de las intervenciones, y el segundo es ver el tipo de ejercicio que se adapta mejor al contexto según ubicación (lugar cerrado) y temperatura (baja). Considerando estos tres datos se recomendaría un tipo de ejercicio como "pausa activa" de máximo 20 minutos para calentar el cuerpo. Paralelamente se deben considerar los factores de riesgo, es decir si la persona fuma, tiene alguna discapacidad, etc. Con este procedimiento es posible lograr una intervención personalizada que no afecte de manera negativa al usuario, sino que promueva hábitos y estilos de vida saludables, por medio de ejercicio y nutrición saludable. Además, el contenido es desplegado con base en el contexto digital de cada usuario, dado que las dimensiones de pantalla y forma de interacción varían entre los dispositivos. 


\subsection{Implementación del modelo de contexto}

\subsubsection{Tecnologías}

Siguiendo algunos lineamientos de la metodología Delphi, se caracterizaron las tecnologías a usar para la implementación del modelo de contexto. Las técnicas usadas fueron consulta bibliográfica, validación con expertos y comparación entre atributos de cada tecnología. Las tecnologías se clasifican en a) tecnologías de sensado del contexto, b) tecnologías móviles.

\section{a) Tecnologías para sensar el contexto}

Para automatizar el proceso de recolección de datos, se debe contar con dispositivos y tecnologías de sensado de información del contexto. En este artículo se seleccionaron las tecnologías que mayores beneficios traen para la salud del usuario, su facilidad de uso y menor complejidad en la implementación del sistema.

Tabla 3. Tecnologías de sensado

\begin{tabular}{|c|c|c|c|c|c|c|c|c|}
\hline & & \multicolumn{4}{|c|}{ Localización } & \multirow[b]{2}{*}{$\begin{array}{c}\text { Complejidad } \\
\text { para sensar } \\
\text { personas } \\
\text { cercanas }\end{array}$} & \multirow[b]{2}{*}{$\begin{array}{c}\text { Interferen- } \\
\text { cia de acti- } \\
\text { vidad según } \\
\text { ubicación }\end{array}$} & \multirow[b]{2}{*}{$\begin{array}{l}\text { Consumo } \\
\text { promedio } \\
\text { de energía } \\
\text { en móviles }\end{array}$} \\
\hline & $\begin{array}{c}\text { Alcance } \\
(\mathrm{m})\end{array}$ & $\begin{array}{c}\text { Precisión } \\
\text { tipo de } \\
\text { localización }\end{array}$ & Error & $\begin{array}{l}\text { Sistema } \\
\text { invasivo }\end{array}$ & $\begin{array}{c}\text { Sistema } \\
\text { extra (con } \\
\text { respecto a } \\
\text { un móvil) }\end{array}$ & & & \\
\hline Bluetooth & 10 o 100 & $\begin{array}{l}\text { Ubicación } \\
\text { Micro }\end{array}$ & $\begin{array}{l}\text { Depende } \\
\text { del punto de } \\
\text { referencia }\end{array}$ & No & $\begin{array}{c}\text { Sí, } \\
\text { punto de } \\
\text { referencia }\end{array}$ & Media & $\begin{array}{c}\text { Depende } \\
\text { del punto de } \\
\text { referencia }\end{array}$ & 221 \\
\hline Wifi & $100-150$ & $\begin{array}{l}\text { Ubicación } \\
\text { Micro }\end{array}$ & $\begin{array}{l}\text { Depende } \\
\text { del punto de } \\
\text { referencia }\end{array}$ & No & $\begin{array}{c}\text { Sí, } \\
\text { punto de } \\
\text { referencia }\end{array}$ & Alta & $\begin{array}{c}\text { Depende } \\
\text { del punto de } \\
\text { referencia }\end{array}$ & 661 \\
\hline \multirow{5}{*}{$\begin{array}{c}\text { Rfid } \\
\text { 30-300 Khz } \\
\text { (LF) } \\
\text { 3-30 Mhz(HF) } \\
\text { 300 Mhz -3 } \\
\text { ghz (UHF) } \\
\text { 2-30 Ghz } \\
\text { (microondas) }\end{array}$} & $0-100$ & \multirow{5}{*}{\begin{tabular}{|c|} 
Ubicación \\
Micro, se- \\
gún tamaño \\
del espacio \\
donde se \\
encuentra el \\
lector
\end{tabular}} & \multirow{5}{*}{$\begin{array}{c}\text { Depende del } \\
\text { usuario }\end{array}$} & \multirow{5}{*}{ Sí } & \multirow{5}{*}{$\begin{array}{l}\text { Sí, dispo- } \\
\text { sitivo del } \\
\text { sistema }\end{array}$} & \multirow{5}{*}{ Baja } & \multirow{5}{*}{ Precisa } & \multirow{5}{*}{$\begin{array}{c}\text { No } \\
\text { soportado }\end{array}$} \\
\hline & $<1$ & & & & & & & \\
\hline & $<1.5$ & & & & & & & \\
\hline & $<100$ & & & & & & & \\
\hline & $<15$ & & & & & & & \\
\hline NFC & Contacto & $\begin{array}{l}\text { Ubicación } \\
\text { situacional } \\
\text { (según } \\
\text { actividad) }\end{array}$ & $\begin{array}{c}\text { Depende del } \\
\text { usuario }\end{array}$ & No & $\begin{array}{l}\text { Sí, los tags } \\
\text { o etiquetas }\end{array}$ & Baja & Precisa & $\begin{array}{c}\approx 55,5 \\
\text { (Consumo } \\
\text { corriente } \\
15 \mathrm{ma} \text { ) }\end{array}$ \\
\hline
\end{tabular}




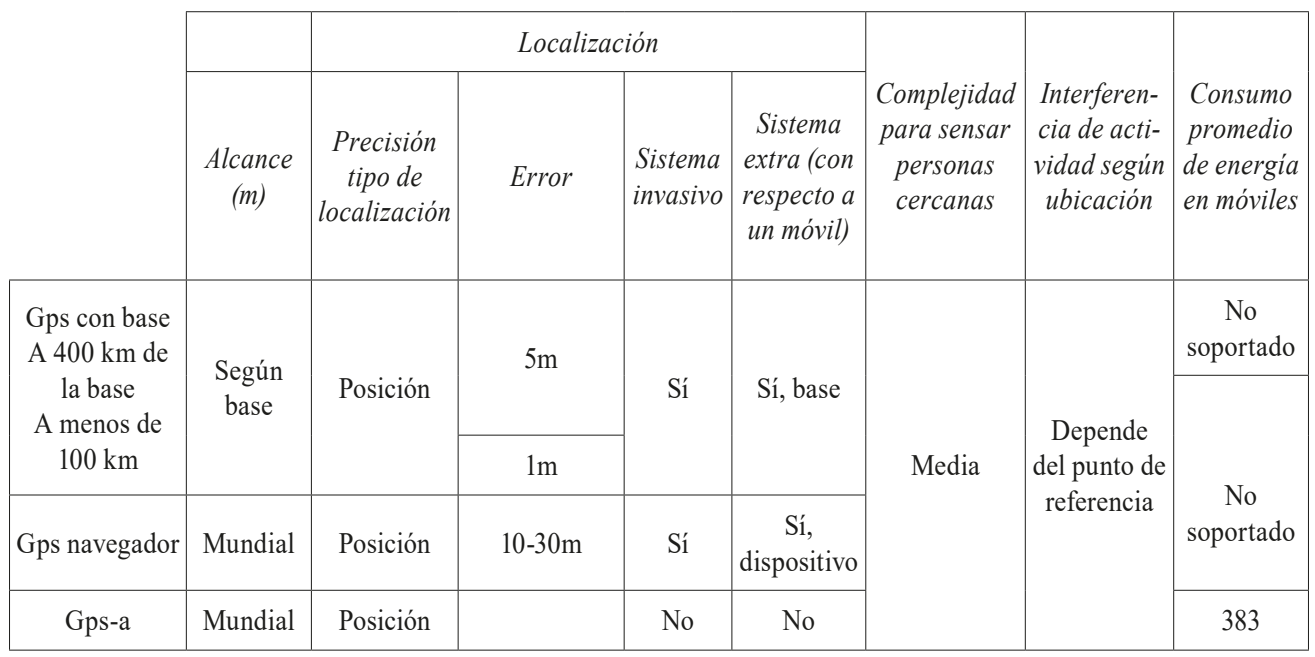

Fuente: elaboración propia

Las tecnologías que permiten sensar algunos de los datos mencionados en la tabla 1, son: GPS, RFID, NFC, Bluetooth y Wi-Fi. Al realizar la comparación entre estas tecnologías, como muestra la tabla 3 se concluyó que:

- Para obtener una localización general del usuario (por ejemplo, si está en: casa, universidad, trabajo o centro comercial) la tecnología que mejor se adapta es GPS [24], debido a su alto grado de exactitud y a su flexibilidad de manejo en aplicaciones móviles.

- Para la localización específica, es decir, en interiores, se determinó que la mejor tecnología para geolocalización en estos espacios (por ejemplo: dormitorio, cocina, puesto de trabajo en oficina, etc.) es NFC (Near Field Communication) [25].

- Para sensar personas cercanas, se seleccionó NFC, ya que a través del uso de etiquetas podrá tomar el ID de usuario y saber quién está cerca. Esto permite al sistema recomendar intervenciones TIC colaborativas que son de gran apoyo en la promoción de actividad física, de manera que los usuarios no realicen el ejercicio de forma individual, sino grupal, según lo requiera.

\section{b) Tecnologías móviles}

Actualmente hay varias alternativas tecnológicas para soportar las intervenciones TIC, como aplicación de escritorio, aplicación web, aplicación para móviles o dispositivo independiente. Con base en la literatura y las recomendaciones de expertos en el tema, los dispositivos móviles son la mejor opción para implementar un sistema consciente en el contexto. 
Para este tipo de sistemas es recomendable el uso de aplicaciones nativas en comparación con las híbridas y las webs, ya que mejoran el diseño y el desempeño del sistema consciente en el contexto. Además, se puede implementar widgets para mostrar datos relevantes o realizar notificaciones aun cuando la aplicación se encuentra cerrada, mejorando así la experiencia del usuario; adicionalmente, las nativas pueden sensar continuamente el contexto en background, permitiendo obtener datos de manera continua y creando una base más sólida con mejor información acerca del contexto del usuario e incluso del propio uso de la aplicación. Por otro lado, se presenta un mejor uso de los recursos del dispositivo y la velocidad de las aplicaciones es mayor. Además, cuenta con NFC y GPS incorporados, es decir, no necesita dispositivos externos de sensado, lo que facilita el uso del sistema.

\subsection{Arquitectura del sistema}

Para el desarrollo del sistema consciente en el contexto se propone la arquitectura mostrada en la figura 5. Se describe a continuación los principales componentes de la misma.

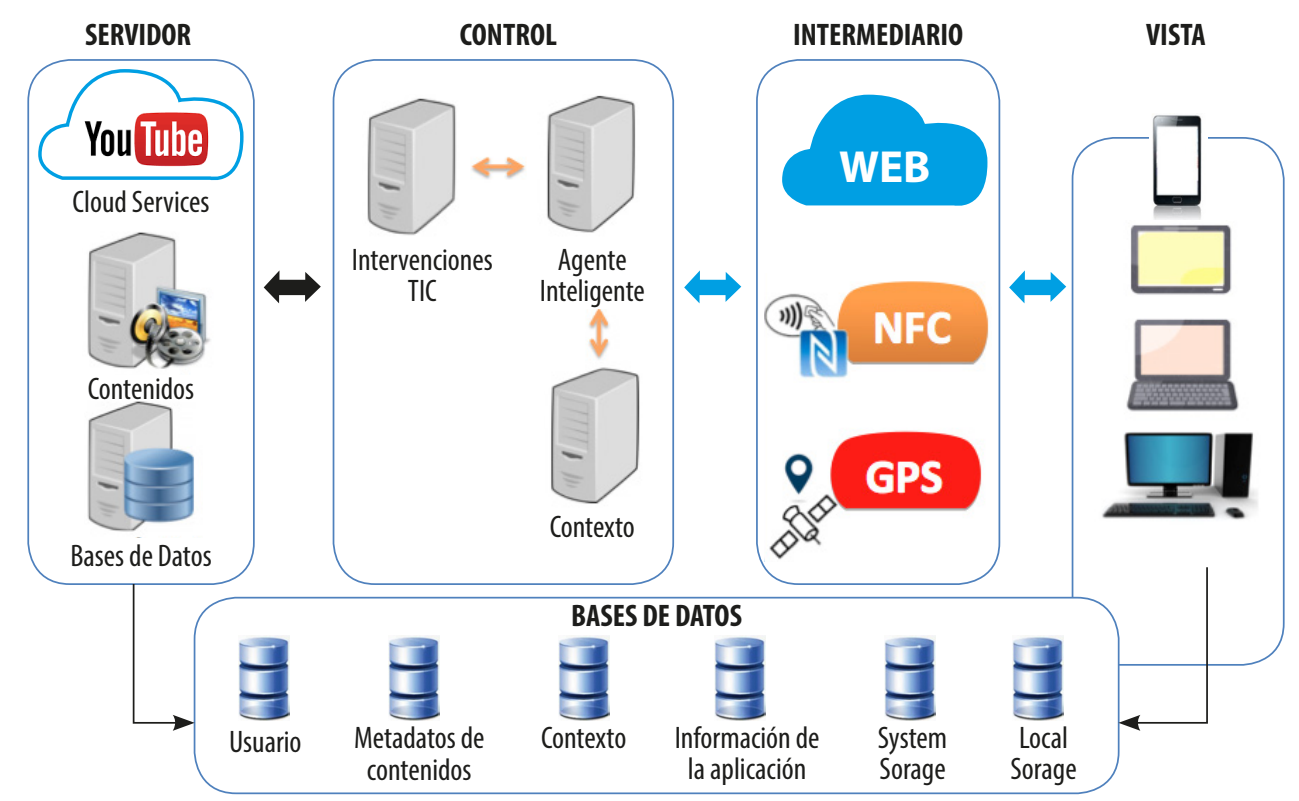

Figura 5: arquitectura de sistema consciente en el contexto.

Fuente: elaboración propia

Cloud services: agrupa las intervenciones TIC que están alojados en servidores online, pertenecen a organizaciones externas al proyecto, como es el caso de "Youtube". 
Este componente se comunica con el servidor de intervenciones TIC para facilitar el recurso multimedia que se asocia a las intervenciones TIC a recomendar al usuario.

Servidor del contexto: su función es recolectar, clasificar y organizar los datos que han sido sensados con NFC y GPS, para localizar espacios cuando no se detectan etiquetas NFC. Estos datos, a su vez, han sido almacenados en la base de datos contexto, y este servidor toma en cuenta los datos que sean relevantes para el contexto de la base de datos del usuario. Estos datos son enviados al servidor agente inteligente.

Servidor agente inteligente: su función es recibir los datos desde el servidor del contexto, procesarlos, realizar inferencias, comunicarse con el servidor de Intervenciones TIC para obtener la clasificación de las intervenciones a recomendar según la información del contexto, del usuario y la inferida. Por último, este componente da la orden para que se desplieguen las intervenciones recomendadas, comunicándose con el servidor de intervenciones TIC. También se comunica con el servidor de base de datos. Además, controla y administrar los datos propios de la aplicación como los datos necesarios para desplegar una interfaz para una persona con discapacidad.

Servidor de contenidos: es un repositorio donde se almacenan intervenciones TIC que están etiquetadas con metadatos, para luego ser enviadas al servidor de intervenciones TIC.

Servidor intervenciones TIC: toma del servidor de contenidos y del cloud services la información sobre las intervenciones TIC y agrupa los contenidos por categorías para después hacer posibles recomendaciones.

Servidor de base de datos: gestiona las bases de datos, permite además el acceso simultáneo de varios servidores o usuarios web; además, coordina la sincronización de los datos en la base de datos offline del local storage (información en la memoria del dispositivo móvil) con las bases de datos generales. El servidor de bases de datos se relaciona con todos los servidores del módulo de control.

\section{Bases de datos}

Usuario: almacena características del usuario que son relevantes para el contexto y para el área de salud, tales como: edad, talla, altura, sexo, peso, entre otros.

Datos de aprendizaje: son datos que el sistema aprende a partir de la experiencia que el usuario ha tenido con el sistema, permitiendo mejorar las futuras recomendaciones de intervenciones TIC a presentar al usuario. Usa formularios, respuestas de feedback del usuario e información relacionada con la aplicación. 
Metadatos de los contenidos: representan la información básica que permite describir y clasificar las intervenciones TIC. Entre los principales metadatos esta: duración, nombre, tipo, URL, para qué tipo de persona sirve, etc.

Contexto: almacenan los datos que se sensaron en el dispositivo móvil como ubicación, actividad, personas cercanas, etc.

System storage: es un backup del local storage; estos datos se han tomado de forma offline, y otra parte son recursos necesarios para el funcionamiento offline, como texto, imágenes, entre otros. Esta base de datos es sincronizada con el Local Storage cuando se tiene acceso a Internet. Su función es servir como punto de comparación entre lo que el móvil almacena y los datos generales del sistema.

Local storage: almacena información en la memoria interna del móvil. Este espacio solo es usado en modo offline, dado que se debe continuar con el funcionamiento de la aplicación aun sin acceso a Internet; posteriormente se sincroniza con el system storage para actualizar información.

\subsection{Desarrollo y pruebas del prototipo}

La arquitectura y los modelos presentados fueron la base para el desarrollo de un primer prototipo del sistema consciente en el contexto para promocionar actividad física y nutrición saludable. Este prototipo aún no tiene implementado un modelo de datos específico para realizar las recomendaciones; sin embargo, cuenta con una lógica adecuada para recomendar, con base en algunas características de contexto, como son: posición, lugar, quién es, con quién está y un tiempo predeterminado.

Este prototipo se evaluó en un grupo focal interdisciplinario conformado así: un estudiante de Educación Media, estudiantes de Ingeniería Electrónica y de Telecomunicaciones de la Universidad del Cauca, un experto en el área de las aplicaciones móviles y profesionales en el área de la salud. A continuación, se describen las principales funcionalidades del prototipo móvil.

Menú de invitado (figura 6): para visualizar este menú no necesita estar registrado; por lo tanto, las recomendaciones son generales y no basadas en datos personales; fue diseñado con el propósito de que los usuarios hagan un recorrido por la aplicación y puedan hacer uso de la misma de una forma básica. En esta primera configuración el usuario puede ver contenidos multimedia de actividad física, nutrición saludable y pausas activas, y guardarlas en favoritos. 


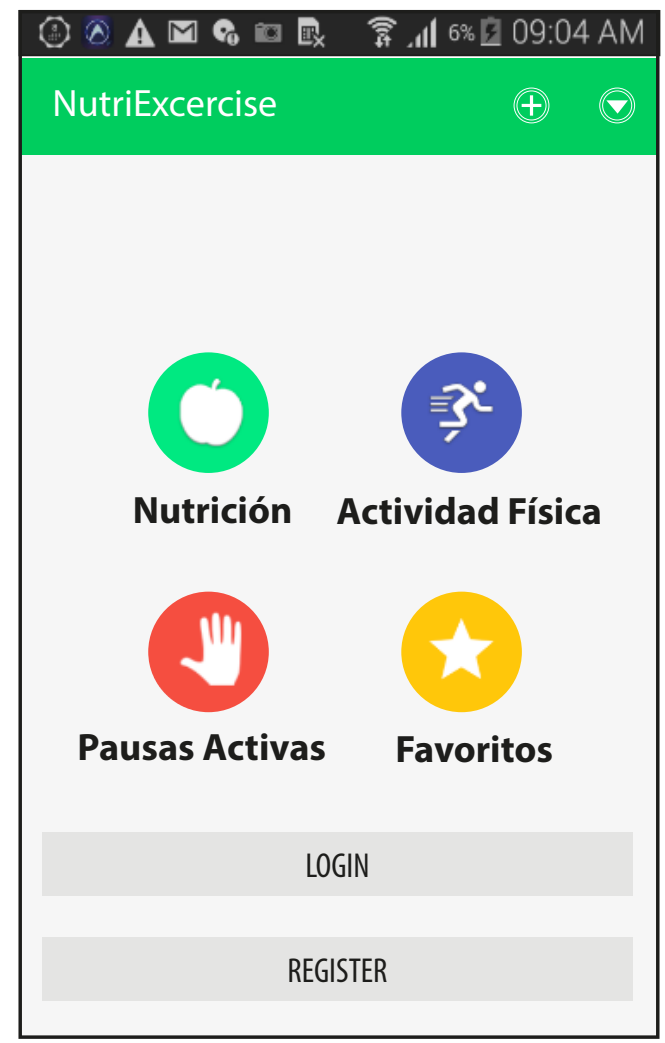

Figura 6. Menú de invitados.

Fuente: elaboración propia

Registro de usuario: en la parte inferior de la figura 7c se observa el botón de registro de usuario; en este punto se obtienen datos esenciales para la personalización como: grupo étnico, si el usuario posee NFC en su celular y horarios habituales de comidas en la mañana, tarde y noche, datos que son almacenados por medio del servidor de bases de datos para que sean almacenados en las bases de datos del contexto y del usuario según corresponda. Después de realizado el registro el usuario procede a realizar su inicio de sesión. En la figura 7 a y 7 b se aprecian dos interfaces del registro y en la figura 7c, la interfaz de inicio de sesión. 


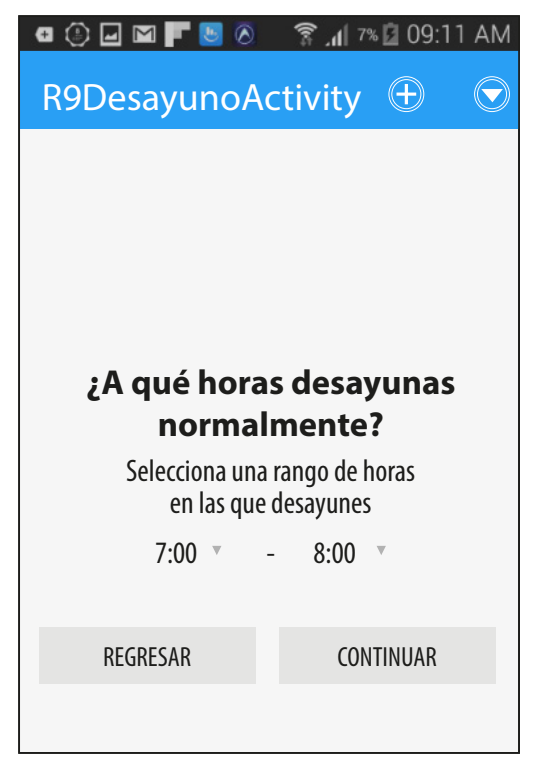

(a) Registro, grupo étnico

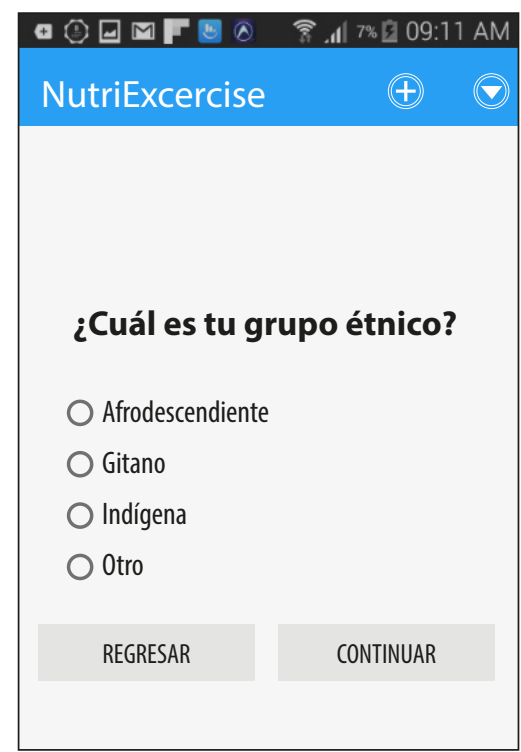

(b) Registro, horario de desayuno

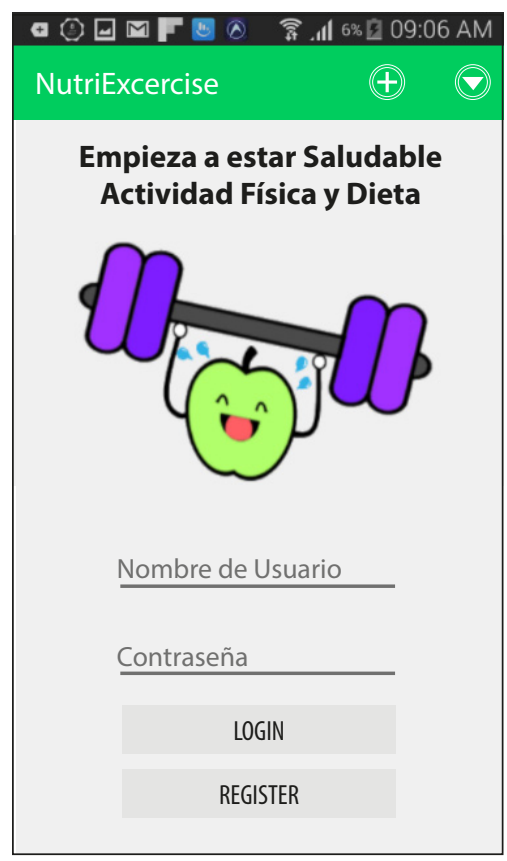

(c) Inicio de sesión

Figura 7. Interfases del registro

Fuente: elaboración propia 
Contexto: una vez el usuario ingresa, el contexto del usuario es identificado por el sistema para realizar las recomendaciones. La información de contexto se guarda en la base de datos del contexto y es procesada desde el módulo de control por el servidor de contexto, en donde según la ubicación del GPS se infiere el lugar en el cual se encuentra el usuario (casa, universidad, trabajo, lugar de recreación), y por medio de NFC se obtiene si el usuario está acompañado y el lugar específico en el que está (cocina, habitación, sala).

Dicha información es entregada por el servidor de contexto al servidor agente inteligente, el cual, a través de un algoritmo de recomendación, toma en cuenta el contexto actual del usuario y los metadatos de las intervenciones existentes en el servidor de intervenciones TIC para decidir las intervenciones más adecuadas.

Menú principal y recomendaciones: cuando el usuario llega al menú después de iniciar sesión, puede escoger una opción del menú principal (figura 8): actividad física, nutrición saludable, pausas activas y favorito, y en el caso de las 3 primeras se obtendrán recomendaciones (figura 9 a y b) basadas en el resultado del algoritmo en el servidor, agente inteligente. Es importante resaltar que el contexto es sensado en todas las interfaces de la aplicación después de realizado el inicio de sesión, para que las intervenciones cambien a medida que el contexto cambie.

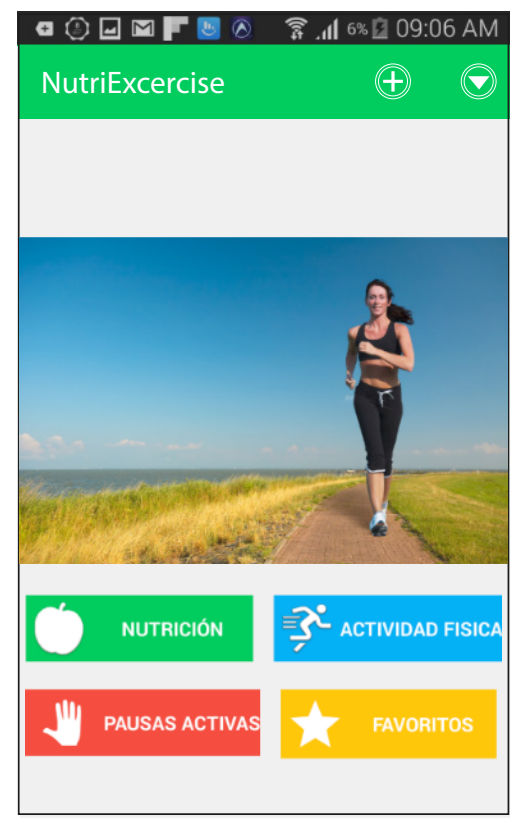

Figura 8. Menú principal.

Fuente: elaboración propia 


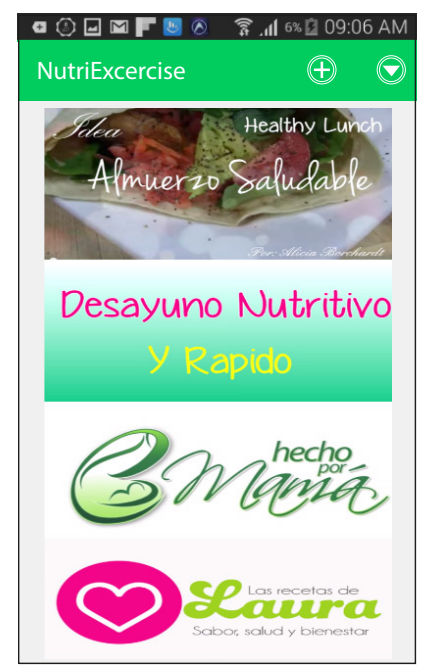

Figura 9. (a) Actividad física

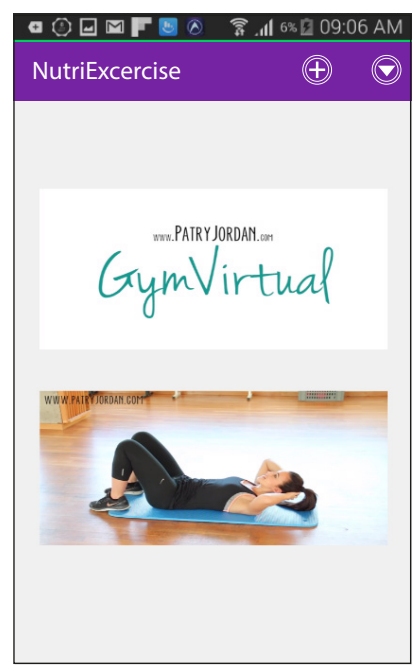

(b) Nutrición saludable

Fuente: elaboración propia

Pausas activas: por otro lado también se la brinda al usuario una opción de acceso directo a las pausas activas (figura 10), dado que con solo sacudir en cualquier dirección su móvil, al detectarse la acción por medio del acelerómetro se le reorientará a las intervenciones TIC de pausas activas; esto con el fin de que el usuario realice pausas activas de forma frecuente para disminuir sensación de fatiga corporal y visual, reducir la tensión muscular y prevenir lesiones como los espasmos musculares, causados por posturas prolongadas y movimientos repetitivos.

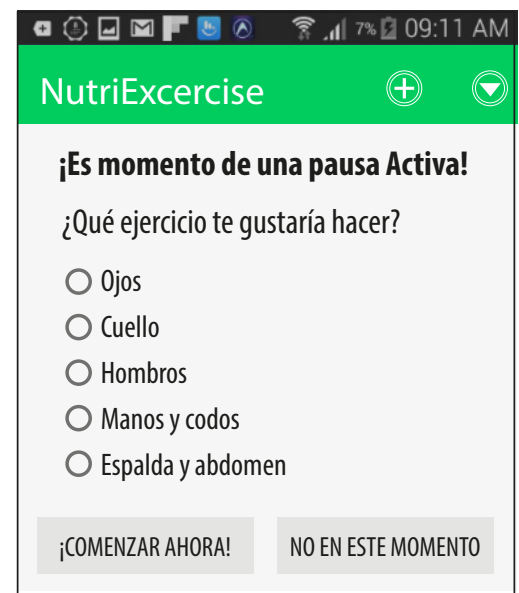

Figura 10. Pausas activas.

Fuente: elaboración propia 
Por último, se realizaron pruebas de usabilidad con este prototipo. En las primeras pruebas se pudo observar que la aplicación no resultaba ser muy intuitiva, porque los usuarios no estaban familiarizados con las tecnologías como NFC y no sabían cómo debían ser utilizados los tags. Por otro lado, el registro era muy largo y era complicado llenar todos los campos porque no se podía deslizar la pantalla y al ser activado el teclado tapaba los campos inferiores; igualmente al realizar el login el teclado tapaba el campo de la contraseña; estos aspectos hicieron que los usuarios tardaran más tiempo en interactuar con la aplicación y en descubrir su funcionamiento. En cuanto a las recomendaciones, se observó que fueron mejor interpretadas las que trataban con el usuario directamente e incentivaban a hacer las actividades junto con el vídeo presentado. Luego, de acuerdo con estas observaciones, se mejoró el prototipo con interfaces amigables y facilidad de uso, lo cual se comprobó en las pruebas siguientes donde se observó que la respuesta de las personas ante la aplicación y las recomendaciones fueron mejores porque: al poner el registro en varias pantallas se facilitó el ingreso a la aplicación; lo que inicialmente tardaba 4 minutos ahora fue aproximadamente 2 minutos dado que el registro tenía varios campos. En la interfaz del login, se logró que los dos campos (usuario y contraseña) estuvieran siempre visibles con lo cual en esta ocasión el login les tardó tan solo 7 segundos, 8 segundos de diferencia respecto a la primera prueba. Para la parte de NFC se mostraron imágenes ilustrativas sobre cómo utilizar los tags, con lo cual se logró que accedieran más rápido a las recomendaciones: pasaron de tardar 45 segundos en poner los tags a tardar tan solo 5 segundos. Se procuró que en todos los vídeos de las recomendaciones se tratara de una forma más directa con el usuario con lo cual se notó una mejoría de respuesta siguiendo las intervenciones en un menor tiempo de aproximadamente 8 segundos, 10 segundos menos que en la primera prueba, cumpliendo con las rutinas recomendadas, siguiendo los consejos y realizando las actividades de acuerdo a como se planteaba en el vídeo sín necesidad de que hubiera intervención de parte de algún experto en la aplicación.

\section{DISCUSIÓN Y CONCLUSIONES}

En este artículo se describió el proceso de diseño de un modelo de contexto para un sistema personalizado de apoyo a la promoción de actividad física y nutrición saludable. El sistema tuvo como finalidad recomendar directamente al usuario, diferentes intervenciones TIC para actividad física y nutrición saludable, que han sido evaluadas previamente por profesionales en salud. Estas recomendaciones se realizarán con base en las características de cada usuario, específicamente su contexto. Un análisis de los resultados y las principales conclusiones se presenta a continuación:

Los datos que se incluyan en un sistema consciente en el contexto para salud deberán ser relacionados con los cuatro factores del contexto (ver figura 2): el contexto 
digital, personal y físico del usuario, además de los factores de riesgo en salud y su influencia en este contexto. De esta forma se obtiene la lista de ítems para la construcción del modelo del contexto, permitiendo clasificar de forma idónea los datos del contexto para el área de salud. Para facilitar la construcción de cualquier modelo de contexto en salud de un sistema personalizado, se recomienda el marco organizado de la figura 3 , que proporciona una serie de etapas interdependientes para modelar un contexto en salud. Este inicia con la identificación de los datos del contexto, su captura, personalización del sistema, hasta el diseño de interfaces de respuesta. El modelo de contexto es fundamental en la implementación de un sistema consciente en el contexto porque menciona los datos del contexto que el sistema debe capturar, sus relaciones y la respuesta que debe generar el sistema de acuerdo con estas. Con base en los modelos de la sección de tecnologías para sensar el contexto antes mencionado, se propone un modelo de contexto, relacionando los datos encontrados y generando recomendaciones con base en estos datos.

Se concluye que es recomendable usar una aplicación nativa para sistemas conscientes del contexto en salud, dado que posee un mayor acceso al dispositivo, en particular a procesos en background y a widgets, además de brindar una mejor experiencia de usuario y mejor desempeño.

La mejor forma (costo efectividad) de sensar datos del contexto es integrar las tecnologías GPS y NFC desde un dispositivo móvil, dado que permiten conseguir los datos del contexto, con un gasto energético bajo, de forma no invasiva.

Para promover actividad física y nutrición saludable se recomienda que el sistema consciente en el contexto tenga apoyo en servidores soportados en computadores y servicios en la nueve, pero que, a su vez, tenga la capacidad de funcionar en forma offline.

Las pruebas de usabilidad al prototipo demuestran que el modelo del sistema consciente el contexto es apropiado para realizar la promoción de actividad física y nutrición saludable en las personas; el modelo orienta de forma clara y precisa cómo implementar el sistema personalizado; además, complementa con una adecuada sugerencia de evaluación. Se observó que fueron mejor interpretadas las recomendaciones que estaban dirigidas directamente al usuario e incentivaban a hacer las actividades junto con el vídeo presentado. Sin embargo, algunas personas no cumplieron las recomendaciones adecuadamente o no las entendían con facilidad, presumiblemente debido a que algunos de ellos no estaban familiarizados con las aplicaciones móviles; otros, en cambio, no gustaban mucho de los vídeos presentados y preferían no hacer las pruebas.

Por otro lado, la personalización de acuerdo con el contexto como se ha establecido es bien aceptada por los usuarios, tiene buena acogida y fue bien evaluada. Lo anterior 
debería motivar a los usuarios a tomar buenos hábitos; sin embargo, para poder demostrar una mejora en el cambio de comportamiento de las personas se requieren otros estudios a más largo plazo. Como trabajo futuro se plantea implementar un prototipo del sistema consciente en el contexto para promoción de actividad física y dieta saludable considerando las recomendaciones de las pruebas de usabilidad, y evaluando el sistema con personas en riesgo de adquirir enfermedades cardiovasculares. También se plantea el modelado de los datos del sistema usando minería de datos.

\section{AGRADECIMIENTOS}

Este trabajo fue realizado en el marco del trabajo de doctorado "Sistema Adaptativo Como Apoyo a la Promoción de Actividad Física y Alimentación Saludable", financiado por Colciencias - Programa de Doctorados Nacionales Convocatoria 617 del 2014.

\section{REFERENCIAS}

[1] WHO, "Informe sobre la situación mundial de las enfermedades no transmisibles 2014", [En línea], Organización Mundial de la Salud, acceso 11 de junio, 2015; Disponible: http://apps. who.int/iris/bitstream/10665/149296/1/WHO_NMH_NVI_15.1_spa.pdf?ua=1\&ua=1.

[2] WHO, "Global action plan for the prevention and control of NCDs 2013-2020", [En línea], Organización Mundial de la Salud, pp. 55, acceso 11 de junio, 2015; Disponible: http://www. who.int/nmh/publications/ncd-action-plan/en/

[3] WHO, "Promoción de la salud y modos de vida sanos," en Resoluciones y Desiciones, Octava sesión plenaria, pp. 37-39, 22 de mayo de 2004 - Comisión A, tercer informe

[4] M, Barrera Perdomo, "Alimentación y nutrición en dislipidemias, síndrome metabólico y enfermedad cardiovascular,” Salud Pública Y Nutrición Humana, pp. 240, 2015.

[5] M. P. Cugelman B1, "Online interventions for social marketing health behavior change campaigns: a meta-analysis of psychological architectures and adherence factors," J Med Internet Res, vol. 1, p. 13, 2011.

[6] G. Cerón Ríos, "Sistema Adaptativo para el Apoyo a Programas de Promoción de Actividad física y Alimentación saludable," Anteproyecto de Tesis de Doctorado, Universidad del Cauca, Popayán, Colombia. Diciembre 2014.

[7] T. Lovett, "Sensing and Interactive Intelligence in Mobile Context Aware Systems," p. 290, Diciembre 2012.

[8] D. ANIND K, "Understanding and Using Context," Personal and Ubiquitous Computing, 2001.

[9] J. M. Gómez Pérez, M. Grobelnik, C. Ruiz, M. Tilly y P. Warren, "Using Task Context to Achieve Effective Information Delivery,” p. 6, 2009. 
[10] B. N. Schilit, N. Adams y R. Want, "Context-Aware Computing Applications," Mobile Computing Systems and Applications, pp. 85-90, 1994.

[11] N. Bricon-Souf y C. R. Newman, "Context awareness in health care: A review," international journal of medical informatics, vol. 76, n. ${ }^{\circ}$ 1, pp. 2-12, 2007.

[12] C. Mettouris, y G. Papadopoulos Contextual, "Modelling in Context-Aware Recommender Systems: A Generic Approach,” Web Information Systems Engineering-WISE 2011 and 2012 Workshops, Springer Berlin Heidelberg, 2013, pp. 41-52

[13] M. Alhamid, "Towards Context-Aware Personalized Recommendations in an Ambient Intelligence Environment”, Tesis Doctoral, Universidad of Ottawa, 2015

[14] F. Buttussi y L. Chittaro, "MOPET: a context-aware and user-adaptive wearable system for fitness training," Artif Intell Med, vol. 42, 2008.

[15] Shih, Chia-Hung, Wakabayashi, N, Yamamura, S, Chen y C.Y, "A context model with a time-dependent multi-layer exception handling policy," International Journal of Innovative Computing, Information and Control, vol. 7, n. ${ }^{\circ}$ 5A, pp. 2225-2234, 2011.

[16] “Engineering Research Methodology,” DigitalOfficePro, 2015. [En línea]. Disponible: http:// www.slideserve.com/donnan/engineering-research-methodology. [Último acceso: 17 marzo 2015].

[17] E. Astigarra, “Método Delphi,” Universidad Deusto, 2011.

[18] R. Galeano, “Diseño centrado en el usuario,” Revista q, vol. 2, n. o 4, pp. 1-15, 2008.

[19] H. Ailisto, P. Alahuhta, V. Haataja, V. Kyllönen y M. Lindholm, "Structuring Context Aware Applications: Five-Layer Model and Example Case,” HJA, vol. 0, n. ${ }^{\circ}$ 11, p. 5, agosto 2002.

[20] C. Bauer y S. Spiekermann, "Conceptualizing context for adaptive pervasive commerce," European Conference on Information Systems (ECIS), p. 13, octubre 2011.

[21] O. S. M. K.-P. C. S. S. N. Salma Najar, "Semantic Representation of Context Models: A Framework for analyzing and understanding," N/P, vol. 1, p. 10, octubre 2009.

[22] “Organización Mundial de la Salud,” OMS [En línea]. Disponible: http://www.who.int/topics/ risk_factors/es/, 2015 [Último acceso: 20 marzo 2015].

[23] O. Coutand, “A Framework for Contextual Personalized Applications,” Kassel University Press GmbH, noviembre 2008.

[24] R. A. Rodriguez, P. M. Vera, M. R. Martínez, D. A. Giulianelli y F. E. Valles, “Context Aware Applications on Mobile Environments-Engaged by the use of NFC," 2013.

[25] "About the Technology, NFC and Contactless Technologies," NFC FORUM, [En línea]. Disponible: http://nfc-forum.org/what-is-nfc/about-the-technology/, 2015, [Último acceso: 23 marzo 2015]. 